\title{
Pseudohypoaldosteronism Type 1-An Exercise in Clinical Deduction and Critical Management
}

\author{
Meenakshi Girish ${ }^{1}$ Pradeep Pazare ${ }^{1}$ Archana Jaiswal ${ }^{1}$ Yash Banait ${ }^{1} \quad$ Richa Kumar $^{1}$ \\ ${ }^{1}$ Department of Pediatrics, NKP Salve Institute of Medical Sciences \& \\ Address for correspondence Meenakshi Girish, MD, 101, Shubham \\ Research Center, Nagpur, Maharashtra, India \\ Enclave, Darda Marg, Rahate Colony, Nagpur 440022, Maharashtra, \\ India (e-mail: meenakshimgirish@gmail.com).
} J Child Sci 2017;7:e127-e129.

\author{
Abstract \\ Keywords \\ - pseudohypoaldo- \\ steronism type 1 \\ - hyperkalemia \\ - acute management \\ - long-term \\ management \\ - autosomal recessive
}

Pseudohypoaldosteronism type 1 (PHA1) is a life-threatening disorder for two reasons, first because it causes severe hyperkalemia and second because the rarity of the disorder means that diagnosis is often delayed due to the lack of clinician familiarity with this condition. In this case report, we have described how even to an unsuspecting mind and eye, a systematic approach can lead to reversal of the severe hyperkalemia and arrive at the diagnosis of PHA1 as a cause of hyperkalemia. Long-term management can be successful only with dedicated care, and the prognosis is unfortunately worsened by the lack of availability of sodium-K resin in many countries, including India.

\section{Introduction}

Pseudohypoaldosteronism type 1 (PHA1) is a rare condition; due to its rarity, a majority of reports have been in the form of case reports or case series. What makes this entity worth reporting yet again is the lesson it gives on a systematic approach to diagnosis and management of life-threatening hyperkalemia. Long-term management of PHA1 is also tricky, and the experiences of other authors have been found to be highly variable. ${ }^{1}$ We would like to present our experience of managing a newborn with PHA1 and her clinical course.

\section{Case}

A 1-month-old phenotypically female infant presented with:

1. Failure to thrive: weight on admission $2.5 \mathrm{~kg}$ (birth weight of $3.1 \mathrm{~kg})$.

2. Severe jaundice: serum bilirubin done a few hours prior to admission found a total bilirubin of $19.2 \mathrm{mg} / \mathrm{dL}$, indirect bilirubin of $18.4 \mathrm{mg} / \mathrm{dL}$, and direct bilirubin of $0.8 \mathrm{mg} / \mathrm{dL}$.

3. Lethargy: infant was conscious but dull and not feeding well.

The infant was born to consanguineous parents (third cousins). There was a family history of two infants who presented similarly, and who suffered sudden deaths in the neonatal period while undergoing phototherapy for jaundice.
Upon hospitalization, blood was drawn for repeat bilirubin, complete blood count, thyroid function tests, reticulocyte count, glucose-6-phosphate dehydrogenase assay, electrolytes, creatinine, and phototherapy was started. The infant was euglycemic.

Very soon the alert resident noted a wide QRS tachycardia on the monitor. The infant had a palpable pulse but had poor perfusion. The infant was immediately given calcium gluconate $10 \%$ intravenously at a dose of $0.5 \mathrm{~mL} / \mathrm{kg}$ slowly over 5 minutes. The tachycardia reverted and laboratory reports showed that sodium was $115 \mathrm{mEq} / \mathrm{L}$ and potassium was $12 \mathrm{mEq} / \mathrm{L}$. Serum creatinine was $0.6 \mathrm{mg} / \mathrm{dL}$ (95th percentile value at 1 month of age is $0.57 \mathrm{mg} / \mathrm{dL}$ ), mildly elevated for her age, but insufficiently high to explain the severe hyperkalemia. A repeat examination of the infant's external genitalia again revealed a normal phenotypic female with no clitoromegaly, effectively ruling out the more common types of congenital adrenal hyperplasia (CAH). The differential diagnosis was now narrowed down to non-CAH-related adrenal insufficiency and one rare type of $\mathrm{CAH}$, congenital lipoid adrenal hyperplasia.

After immediately starting intravenous sodium bicarbonate and glucose insulin drip for treatment of severe hyperkalemia, a sample was drawn for serum cortisol and intravenous hydrocortisone bolus and infusion was started. Arterial blood gas revealed severe metabolic acidosis, which was treated by titrating the sodium bicarbonate infusion. Over the next received

July 13, 2017

accepted after revision

September 2, 2017
DOI https://doi.org/

10.1055/s-0037-1607237. ISSN 2474-5871.
Copyright (c) 2017 Georg Thieme Verlag License terms

KG Stuttgart · New York 
few hours, serum potassium declined insufficiently, from 12 to $10 \mathrm{mEq} / \mathrm{L}$; therefore, peritoneal dialysis was performed for hyperkalemia.

The serum cortisol level subsequently returned at $39.4 \mu \mathrm{g} /$ $\mathrm{dL}$ (normal range: $2.8-23 \mu \mathrm{g} / \mathrm{dL}$ ), effectively ruling out adrenal insufficiency. The hydrocortisone infusion was stopped, and an early morning blood sample was sent for plasma aldosterone and plasma renin activity levels.

While an intravenous glucose and insulin infusion help decrease the fraction of potassium in the extracellular space, it does not decrease the total potassium content of the body. For this, all potassium intakes should be stopped and potassium-binding resins have to be used. Of the two resins available, the sodium polystyrene resin is preferable, as it helps in correcting the concomitant hyponatremia seen in many of these patients, while the calcium polystyrene resin ( $\mathrm{K}$ bind) has the disadvantage of causing hypercalcemia. We were forced to use the calcium polystyrene resin as the sodium polystyrene resin was unavailable.

For long-term management of hyponatremia, the infant was started on oral $3 \%$ saline $(22 \mathrm{mEq} / \mathrm{d}$ ) and this with the oral sodium bicarbonate tablets helped maintain sodium in the normal range. For control of serum potassium, the infant was started on $\mathrm{K}$ bind (calcium polystyrene resin enema) $24 \mathrm{~g} / \mathrm{d}$ in four to five divided doses with alternate day monitoring of serum potassium. Despite the fact that there is resistance to action of aldosterone at the receptor level, high dose of oral fludrocortisone has been recommended, ${ }^{2}$ and therefore, it was started in this infant at a dose of $1 \mathrm{mg} / \mathrm{d}$.

\section{Diagnosis}

In the absence of hyperkalemia due to increased intake or transcellular shift of potassium which occurs in conditions easily picked up by history alone, disorders of the reninangiotensin-aldosterone axis constitute an important group where hyponatremia is associated with hyperkalemia and simple clinical deduction helped us rule out most of the causes.

- CAH with concomitant mineralocorticoid deficiency is seen in 21 hydroxylase deficiency, $3 \beta$ hydroxysteroid dehydrogenase deficiency, and congenital lipoid hyperplasia. The first two also cause virilization in female neonates. The other conditions causing $\mathrm{CAH}, 11$ hydroxylase deficiency and 17 hydroxylase deficiency, have mineralocorticoid excess and hence lead to hypokalemia rather than hyperkalemia, along with glucocorticoid deficiency.

- Congenital adrenal hypoplasia can be diagnosed by a low serum cortisol.

- Congenital defects in aldosterone synthesis will present biochemically with hyponatremia and hyperkalemia but will have very low aldosterone level and elevations in plasma renin activity.

However, laboratory evaluation revealed markedly elevated aldosterone and renin values: plasma renin activity of $>37.0 \mathrm{ng} / \mathrm{mL} / \mathrm{h}$ (reference range: $1-4 \mathrm{ng} / \mathrm{mL} / \mathrm{h}$ ) and serum aldosterone of $769 \mathrm{ng} / \mathrm{dL}$ (reference range: 7-99 ng/dL), ruling out all of the above possibilities. This leaves us with a very rare condition:

- Disorders of aldosterone resistance or PHA helped us come to the final diagnosis of PHA1.

Genetic studies are planned for confirmation of PHA subtype, subject to availability of financial resources.

\section{Discussion}

This case report provides some important learning points in the diagnosis and management of a complex and often lifethreatening neonatal condition.

- Hyperkalemia management: When confronted with a life-threatening emergency in the form of a cardiac arrhythmia, IV calcium boluses can be lifesaving, as happened in our case.

- Clinically, salt-wasting variants of CAH can be indistinguishable from PHA. Although the former is far more common, the latter should be suspected in a female neonate with normal genitalia in the setting of severe salt wasting and failure to respond to initial hydrocortisone therapy.

PHA1 has two subtypes. Autosomal dominant-type PHA1 (renal PHA1) occurs due to loss of function mutations in the mineralocorticoid receptor gene $N R 3 C 2$ (nuclear receptor subfamily 3 group $C$ member 2), and hence is restricted to renal salt wasting and is reported to improve with age, ${ }^{3}$ autosomal recessive type (systemic PHA1) occurs due to a loss of function mutation in the epithelial sodium channel. This channel, which is regulated by aldosterone, comprised an $\alpha$ subunit, $\beta$ subunit, and $\gamma$ subunit, respectively, encoded by the genes SCNN1A, SCNN1B, and SCNN1G. Autosomal recessive PHA1 occurs due to mutations in SCNN1G. As this channel is expressed in multiple organs (including the distal nephron), manifestations are due to involvement of several target organs, including the colon, salivary glands, and sweat glands in addition to the kidney. Skin changes and increased respiratory infections have been reported, and the disease tends to persist into adult life. ${ }^{4}$ In view of the poor genotypephenotype correlation reported in PHA $1,{ }^{5}$ it is difficult to prognosticate from the clinical course alone. Our case had a stormy neonatal course with a definite history or mortality in two siblings, and with clinically asymptomatic parents with distant consanguinity, suggesting an autosomal recessive inheritance. However, this infant did not manifest any skin lesions as reported in the literature ${ }^{1,6}$ and has not yet developed any respiratory infections at the time of submission. The infant was breastfed and weight gain has improved at 3.5 months of age, the infant's weight is now $4 \mathrm{~kg}$, still well below the 5th weight percentile on the WHO curve but improved considerably from the time of presentation.

\section{Conclusion}

PHA1 is unique not just because of its rarity but also because it demands a high degree of clinical acumen in diagnosis as 
well as management. From management for acute lifethreatening arrhythmia to drug manipulations for longterm control of electrolytes, this case is a lesson in clinical deduction and algorithmic management of hyperkalemia.

\section{Authors' Contributions}

M.G. and R.K. were responsible for case management and writing the article, Y.B. helped in reviewing the literature and discussion, and P.P. and A.J. helped in critical revision. All authors contributed toward final approval of article and take responsibility for accuracy and integrity of the work.

Funding

None.

\section{Conflict of Interest}

None.

\section{References}

1 Amin N, Alvi NS, Barth JH, et al. Pseudohypoaldosteronism type 1: clinical features and management in infancy. Endocrinol Diabetes Metab Case Rep 2013;2013:130010

2 Arai K, Tsigos C, Suzuki Y, et al. Physiological and molecular aspects of mineralocorticoid receptor action in pseudohypoaldosteronism: a responsiveness test and therapy. J Clin Endocrinol Metab 1994;79(04):1019-1023

3 Young WF. Etiology, diagnosis, and treatment of hypoaldosteronism (type 4 RTA). In: UpToDate, Post TW (Ed), UpToDate, Waltham, MA. (Accessed on June 21, 2017.)

4 Enuka Y, Hanukoglu I, Edelheit O, Vaknine H, Hanukoglu A. Epithelial sodium channels $(\mathrm{ENaC})$ are uniformly distributed on motile cilia in the oviduct and the respiratory airways. Histochem Cell Biol 2012;137(03):339-353

5 Attia NA, Marzouk YI. Pseudohypoaldosteronism in a neonate presenting as life-threatening hyperkalemia. Case Rep Endocrinol 2016;2016:6384697

6 Sharma R, Pandey M, Kanwal SK, Zennaro MC. Pseudohypoaldosteronism type 1: management issues. Indian Pediatr 2013;50(03): 331-333 